\title{
FORMULATION OF MULBERRY LEAF (MORUS ALBA L.) EXTRACT HYDROGEL BEADS USING CROSS-LINKED PECTIN
}

\section{SILVIA SURINI*, DEFIRA METHA DIANDRA}

\author{
Department of Pharmacy, Faculty of Pharmacy, Universitas Indonesia, Jakarta, Indonesia. Email: silvia@farmasi.ui.ac.id
}

Received: 21 April 2017, Revised and Accepted: 18 July 2017

\section{ABSTRACT}

Objective: Nutraceutical products have now gained considerable interest to be consumed regularly. Since the active substances in nutraceutical products are provided by nature, they are safe to consume. The development of a dosage form and the appearance of a modern nutraceutical product can improve product quality. Therefore, this research aimed to formulate a nutraceutical product, mulberry leaf extract, into hydrogel beads that swell in water and that can be administered orally.

Methods: Deoxynojirimycin, the active substance in mulberry leaf extract hydrogel beads, has the efficacy of an anti-diabetic agent. In this research, there were four formulations: The variations $1: 2$ and $1: 3$ of the extract-to-polymer ratio and the variations $2.5 \%$ and $5 \%$ of the cross-linker concentration.

Results: The results showed that hydrogel beads had a spherical form with an unsmooth surface and a brown color, and they were able to swell in water. The best formula was Formula 1, with a 1:2 extract-to-polymer ratio and a $2.5 \%$ cross-linker concentration. Formula 1 produced an average diameter of $3.6 \mathrm{~mm}$, a swelling ability of $210.68 \%$, and immediate swelling in water for 30 seconds.

Conclusions: Mulberry leaf extract hydrogel beads could be used as an interesting nutraceutical product.

Keywords: Beads, Mulberry leaf extract, Pectin, Zinc acetate.

(C) 2017 The Authors. Published by Innovare Academic Sciences Pvt Ltd. This is an open access article under the CCBY license (http://creativecommons org/licenses/by/4. 0/) DOI: http://dx.doi.org/10.22159/ijap.2017.v9s1.78_85

\section{INTRODUCTION}

Nowadays, nutraceutical products have been widely developed to prevent and treat disease, as well as to maintain public health. A 19992000 National Health and Nutrition Examination Survey showed that more than 5,000 of the 10,000 respondents consumed a nutraceutical product regularly. Nutraceutical products can be food or food-related, such as dietary supplements, herbal products, cereals, soups, and healthy drinks. The therapeutic areas covered by nutraceutical products include cancers, osteoporosis, hypertension, cholesterol, depression, and diabetes [1-3]. A previous study showed that mulberry leaf extract had an anti-diabetic effect in diabetes-induced Wistar rats [4,5]. Mulberry leaves (Morus alba L.) contain 1-deoxynojirimycin and its derivatives, an intestinal glucosidase inhibitor that prevents the hydrolysis of carbohydrates to monosaccharide, and therefore, suppresses the blood glucose level [6]. Mulberry leaf extract has been formulated as an effervescent tablet, whitening cream, and capsule.

Beads are a spherical dosage form containing an active substance. They can enhance the bioavailability and stability of drugs, provide a uniform distribution of drugs, and have an interesting appearance as a dosage form. Hydrogel beads are able to swell when in water because the hydrophilic polymer of the hydrogel is crosslinked with its cross linker. Ionic gelation is the method used to cross-link polymer and its cross linker to produce hydrogel beads.

Hydrogel beads can be made from natural and synthetic polymers. Much research efforts have been concentrated on the development of hydrogel beads using natural polymers, as they have good solubility in water, a high swelling ability, a low toxicity level, and high biodegradability and biocompatibility. Pectin as a natural polymer to produce beads has a bioactive substance that is able to suppress blood glucose through an inhibition mechanism that prevents the absorption of glucose into the blood [7]. Zinc is a cation divalent that is cross linked with the carboxyl group of pectin to create hydrogel beads. A study reported that zinc possesses a high ability to bind with pectin, which can form beads with a greater gel strength [8]. This research aims to make and evaluate mulberry leaf extract hydrogel beads with pectin as a polymer and zinc acetate as a cross linker to produce a nutraceutical product in a bead dosage form.

\section{MATERIALS AND METHODS}

Materials

Dry mulberry leaf extract (Huamao Biotechnology, Yongzhou, China), mulberry leaf extract (Balitro, Bogor, Indonesia), pectin (Danisco, Grindsted, Denmark), zinc acetate (Merck, Darmstadt, Germany), and distilled water (Brataco, Jakarta, Indonesia) were purchased.

\section{Equipment}

An EB-330 analytical balance (Shimizu, Kyoto, Japan), magnetic stirrer (IKA, Staufen, Germany), freeze dryer (Vacuubrand, Wertheim, Germany), Nikon model Eclipse E 200 optical microscope (Nikon model Eclipse E 200, Tokyo, Japan), AMB 50 moisture analyzer (Adam, Connecticut, United States), XT2i texture analyzer (Stable Micro System, Godalming, England), Vernier caliper (Tricle Brand, Shanghai, China), pH meter type 510 (Eutech Instrument, Singapore, Singapore), and Brookfield viscometer (Brookfield, Wisconsin, United States) were used.

\section{Methods}

To ensure optimum conditions, formula optimization was conducted beforehand. Five parameters have been optimized, as shown in Table 1.

The formula of the beads can be seen in Table 2 with variations in zinc acetate and pectin concentrations.

Mulberry leaf extract hydrogel beads were prepared by the ionic gelation method. Pectin and mulberry leaf extracts were solubilized in distilled water and then mixed until they became homogenous. After that, the mixture of mulberry extract and pectin was dropped into the zinc acetate solution by a hose with a diameter of $2.5 \mathrm{~mm}$. The crosslinking time was 15 minutes with mechanical stirring, after which the 
beads were filtered and washed with distilled water. Finally, the beads were dried using a freeze dryer.

\section{Evaluations of mulberry leaf extract hydrogel beads \\ Yield}

The rendemen was calculated using the following equation:

$\mathrm{Wp}=\frac{\mathrm{Wm}}{\mathrm{Wt}} \times 100 \%$

Where $\mathrm{Wp}$ is the yield, Wm is the total weight of the beads after formulation, and Wt is the total weight of beads being prepared before formulation.

\section{Shape and morphology}

An optical microscope was used for the shape and morphology examination.

\section{Particle size distribution}

The diameters of 300 randomly selected beads from each formula were measured using a Vernier caliper

\section{Swelling ability}

The 100-mg beads from each formula were placed in weighing dishes. After, $10 \mathrm{~mL}$ of distilled water was added. Then, the swelling of the beads in room temperature was observed. Beads were weighed using an analytical balance at 5, 10, 15, 30, 60, 90, and 120 minutes, and swelling ability was calculated by the following equation:

Swelling ability $(\%)=\frac{\mathrm{W} 2-\mathrm{W} 1}{\mathrm{~W} 1} \times 100 \%$

\section{Swelling time}

The $10 \mathrm{~mL}$ of distilled water was added to the 100-mg beads from each formula were placed in weighing dishes. Then, the swelling of the beads was observed in terms of time.

\section{Moisture content}

Moisture content was determined using a moisture analyzer. The equipment was prepared to reach a temperature of $105^{\circ} \mathrm{C}$ before use. Then, 1-g beads were spread on a plate, and the moisture analyzer was turned on to show the beads' moisture content.

\section{Gel strength}

The gel strength of hydrogel beads was measured using a texture analyzer. Samples were placed on a plate, and the probe was pressed to the sample to determine the gel strength of each sample.

\section{RESULTS AND DISCUSSION}

\section{Optimization}

To ensure optimum conditions, formula optimization was conducted beforehand. The optimization results of the cross-linker concentration were $2.5 \%$ and $5 \%$ because those concentrations created spherical beads with no tail. The shape of the spherical beads was caused by the process of crosslinking between pectin and zinc acetate. The dry extract was chosen as the best kind of extract to formulate hydrogel beads because it makes attractive beads with a brown color, whereas the wet extract makes dark-green beads with black freckles. The best extract and polymer ratios were $1: 2$ and 1:3 because the mixture of the extract and pectin can flow into a hose and form spherical beads. 15 minutes was chosen as the best cross-linking time because it results in beads having a good swelling ability. The best drying method was freeze-drying because it allowed the beads to retain their same shape and size as before drying, even though there were some deflations on some sides of the beads. The beads before and after the drying process are shown in Fig. 1.

\section{Beads evaluation}

Yield

The results of the yield of mulberry leaf extract hydrogel beads were $82.25 \%, 78.88 \%, 63.30 \%$, and $73.93 \%$ in Formulas 1-4, respectively. From the results, it can be seen that the yield values were in the range of $63.30-82.25 \%$. The results did not reach $100 \%$ due to the loss of distilled water during the drying process using the freeze dryer.

\section{Shape and morphology}

Beads were observed using an optical microscope with $\times 40$ magnification. Beads before the drying process were spherical with unsmooth surfaces. Beads after the drying process had a uniform shape, a light-brown color, and a slightly deflated texture. It could be that the drying mechanism of freeze-drying withdrew $\mathrm{H}_{2} \mathrm{O}$, producing an empty cavity.

\section{Particle size distribution}

The particle size distribution was observed by measuring the diameters of the individual 300 beads for each formula using a Vernier caliper. The results of the measurement of the average diameter of the beads using each formula are shown in Table 3. Table 3 shows the measurements of the average diameter of the beads.

\section{Swelling ability}

The purpose of the swelling ability test is to determine how much and for how long the beads could swell in distilled water, as the beads would be consumed using distilled water by consumers. The swelling ability result is shown in Fig. 2, where it can be seen that each formula was different for each minute measurement. After testing the swelling ability for up to $2 \mathrm{hrs}$, the results were $210.68 \%, 207.43 \%, 255.81 \%$, and $220.75 \%$ for Formulas 1-4, respectively. The result shows that Formula 3 had the greatest swelling ability, whereas Formula 1 had the least swelling ability. This result can be explained by the extractto-polymer ratio of 1:3 in Formula 3, a greater amount of polymers and the crosslinking of hydrogel beads causing the hydrogen bonds became larger. With the great number of hydrogen bonds, the ability to bind with water and the ability to swell in water become greater [9]. When compared with the research from Das et al., the swelling ability of

Table 1: Parameters of formula optimization

\begin{tabular}{ll}
\hline Parameter & Variable \\
\hline Zinc acetate & $2.5 \%, 5 \%(\mathrm{pH} 1.3,3.0$, and 6.3), \\
concentration & $10 \%, 15 \%$ and $20 \%$ \\
Kind of extract & Dry or wet extract \\
Extract to pectin ratio & $1: 1,1: 2$ and $1: 3$ \\
Cross-linking time & $15,30,45,60$ and 120 minutes \\
Drying method & Room temperature and freeze dryer \\
\hline
\end{tabular}

Table 2: Formulation of mulberry leaf extract hydrogel beads

\begin{tabular}{lllll}
\hline Materials & F1 & F2 & F3 & F4 \\
\hline Mulberry leaf extract (g) & 1.2 & 1.2 & 1.2 & 1.2 \\
Pectin (g) & 2.4 & 2.4 & 3.6 & 3.6 \\
Distilled water (mL) & 48.0 & 48.0 & 72.0 & 72.0 \\
Zinc acetate solution (\%) & 2.5 & 5.0 & 2.5 & 5.0 \\
\hline
\end{tabular}

Table 3: Average diameter of mulberry leaf extract hydrogel beads

\begin{tabular}{ll}
\hline Formula & Average diameter of beads $(\mathbf{m m})$ \\
\hline 1 & $3.59 \pm 0.04$ \\
2 & $3.93 \pm 0.11$ \\
3 & $3.55 \pm 0.03$ \\
4 & $3.81 \pm 0.03$ \\
\hline
\end{tabular}



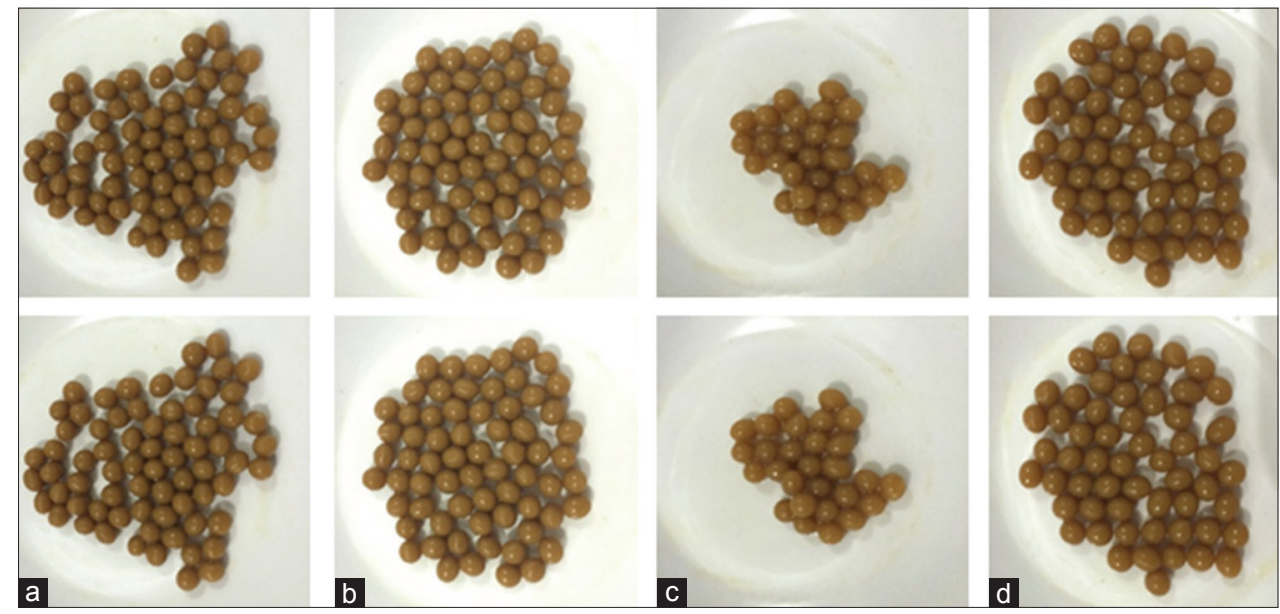

Fig. 1: The appearance of the beads before (1) and after (2) the drying process, (a) Formula 1 (b) Formula 2, (c) Formula 3, and (d) Formula 4

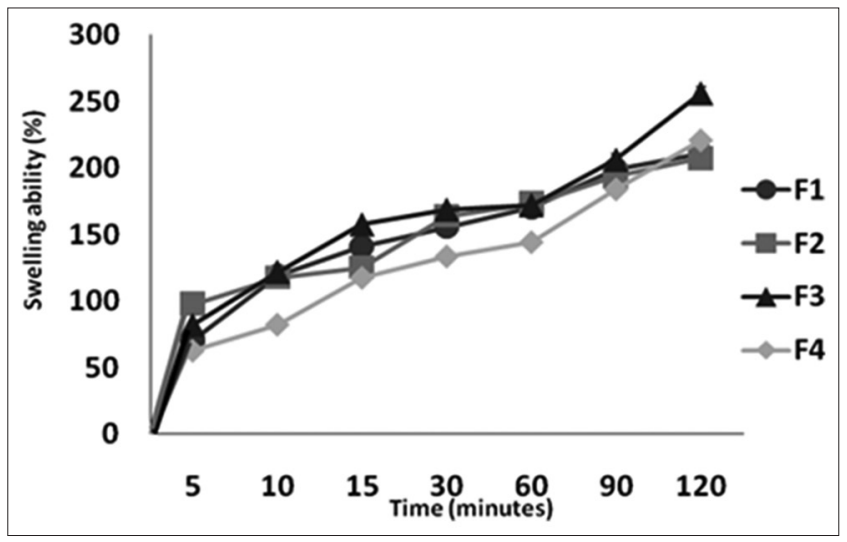

Fig. 2: Swelling ability of mulberry leaf extract hydrogel beads

hydrogel beads is $<100 \%$, whereas the swelling ability of mulberry leaf extract was better, at more than $200 \%$ [8].

\section{Swelling time}

Swelling time was tested by observing the time needed for the beads to swell the first time in distilled water. The swelling time test was done visually by watching the beads as they began to sink into the distilled water from their initial floating position in distilled water.

Table 4 shows the result of the swelling times of mulberry leaf extract hydrogel beads from formulas 1 until 4, respectively. Using Formula 1, the beads first swell in water at $30 \mathrm{~s}$, and they swell at $35 \mathrm{~s}$ using Formula 2, $23 \mathrm{~s}$ using Formula 3, and $16 \mathrm{~s}$ using Formula 4. The fastest swelling time was with Formula 4, while Formula 2 had the slowest swelling time. This was because Formulas 3 and 4 used an extract-topolymer ratio of 1:3. The more polymers cross linked with an extract, the larger the cross-linked hydrogel beads became, so more and more hydrogen bonds were formed. With the great number of hydrogen bonds, the beads swelled and bonded with water faster4. From the results obtained, all formulations had a swelling time of $<1$ minute. This result could be explained by the fact that the cross-linking time was not long (15 minutes), so it caused the beads to swell quickly $[5,6]$. Furthermore, pectin was used as the polymer, and it is a hydrophilic polymer, so it can absorb water.

\section{Moisture content}

The results of the moisture content test are shown in Table 5.

Table 5 shows the results of the test of the moisture content of mulberry leaf extract hydrogel beads, which were $21.09 \%, 26.52 \%, 26.44 \%$, and
Table 4: Swelling time of mulberry leaf extract hydrogel beads

\begin{tabular}{ll}
\hline Formula & Swelling time (s) \\
\hline 1 & $30 \pm 1$ \\
2 & $35 \pm 3$ \\
3 & $23 \pm 2$ \\
4 & $16 \pm 2$ \\
\hline
\end{tabular}

Table 5: Moisture content of mulberry leaf extract hydrogel beads

\begin{tabular}{ll}
\hline Formula & Moisture content (\%) \\
\hline 1 & $21.09 \pm 0.12$ \\
2 & $26.52 \pm 0.50$ \\
3 & $26.44 \pm 0.40$ \\
4 & $23.64 \pm 0.50$ \\
\hline
\end{tabular}

$23.64 \%$ for Formulas 1-4, respectively. This was due to the polymer in the beads, which was pectin, a hydrophilic polymer that can absorb water and high humidity.

\section{Gel strength}

The gel strength test aimed to determine the ability to maintain the shape of the hydrogel beads. Gel strength test results can be seen in Table 6 .

Table 6 shows that the gel strength of the hydrogel beads before drying was stronger than after drying. In all formulations, the gel strength of the beads after drying decreased compared to before drying. However, the decrease was not too large, except in Formula 2, where the gel strength of the beads before drying was $551.4 \mathrm{gf}$, and it was $274.9 \mathrm{gf}$ after drying. The decrease in the gel strength of the dry beads occurred because the beads before drying had a chewy texture and could break if pressed, but the beads after drying had a texture that when pressed, the beads would not only break but also deflate. In Formula 2, the gel strength of the beads was more decreased before to after drying. This could be because the dry beads tested in Formula 2 were deflated, so when the probe was pressed, the beads deflated directly, resulting in lesser gel strength.

\section{CONCLUSION}

The results of this study indicate that the hydrogel beads had spherical shape with an unsmooth surface, were able to swell in water, and were able to maintain their form. In addition, Formula 1 was chosen as the best formula for mulberry leaf extract hydrogel beads to ensure a proper spherical shape and brown color, an average diameter of $3.6 \mathrm{~mm}$, a swelling ability of $210.68 \%$, a swelling time of 30 seconds, and a yield value of $82.25 \%$. 
Table 6: Gel strength of mulberry leaf extract hydrogel beads

\begin{tabular}{lll}
\hline Formula & \multicolumn{2}{l}{ Hardness $(\mathrm{gf})$} \\
\cline { 2 - 3 } & Beads before drying & Beads after drying \\
\hline 1 & 493.1 & 461.3 \\
2 & 551.4 & 274.9 \\
3 & 382.8 & 323.4 \\
4 & 331.6 & 356.7 \\
\hline
\end{tabular}

\section{REFERENCES}

1. Patil JS, Kamalapur MV, Marapur SC, Kadam DV. Ionotropic gelation and polyelectrolyte complexation: The novel techniques to design hydrogel particulate sustained, modulated drug delivery system - A review. Dig J Nanomater Biostruct 2010;5(1):241-8.

2. Shukla Y, George J. Combinatorial strategies employing nutraceuticals for cancer development. Ann N Y Acad Sci 2011;1229:162-75.
3. Nabavi SM, Daglia M, Braidy N, Nabavi SF. Natural products, micronutrients, and nutraceuticals for the treatment of depression: A short review. Nutr Neurosci 2017;20(3):180-94.

4. Mohammadi J, Naik PR. Evaluation of hypoglycemic effect of Morus alba in an animal model. Indian J Pharmacol 2008;40(1):15-8.

5. Wilson RD, Islam MS. Effects of white mulberry (Morus alba) leaf tea investigated in a Type 2 diabetes model of rats. Acta Pol Pharm 2015;72(1):153-60.

6. Kimura T, Nakagawa K, Kubota H, Kojima Y, Goto Y, Yamagishi K, et al. Food-grade mulberry powder enriched with 1-deoxynojirimycine suppresses the elevation of postprandial blood glucose in human. J Agric Food Chem 2007;55(14):5869-74.

7. Maulida D, Estiasih T. Hypoglycaemic effect of gadung tuber (Dioscorea hispida) water soluble polysaccharide and alginate: A review. J Pangan Agroindustri 2014;2(3):136-40.

8. Das $\mathrm{S}, \mathrm{Ng} \mathrm{K}, \mathrm{Ho}$ PC. Formulation and optimization of zinc-pectinate beads for the controlled delivery of resveratrol. AAPS Pharm Sci Tech 2010;11(2):729-42.

9. Maitra J, Shukla VK. Cross-linking in hydrogels - A review. Am J Polym Sci 2014;4(2):25-31. 